Eric F. Morand Grant/research support from: AstraZeneca, Consultant of: AstraZeneca, Speakers bureau: AstraZeneca

DOI: 10.1136/annrheumdis-2020-eular.3007

\section{AB0385 $\quad$ TARGETING CD38 IN SYSTEMIC LUPUS ERYTHEMATOSUS}

L. Ostendorf $^{1,2}$, U. Schneider ${ }^{1}$, M. Urbicht ${ }^{2}$, P. Enghard ${ }^{2,3}$, F. Heinrich ${ }^{2}$, P. Durek ${ }^{2}$, G. Heinz ${ }^{2}$, H. Mei $^{2}$, M. F. Mashreghi², G. R. Burmester ${ }^{1}$, A. Radbruch ${ }^{2}$, F. Hiepe ${ }^{1,2}$, T. Alexander ${ }^{1,2}$. ${ }^{1}$ Charité - Universitätsmedizin Berlin Department of Rheumatology and Clinical Immunology, Berlin, Germany; ${ }^{2}$ Deutsches Rheuma-Forschungszentrum (DRFZ), Berlin, Germany; ${ }^{3}$ Charité Universitätsmedizin Berlin, Department of Nephrology, Berlin, Germany

Background: Depletion of long-lived plasma cells (PC) resembles a novel concept for the treatment of antibody-mediated autoimmune diseases, such as systemic lupus erythematosus (SLE). Therapeutic approaches such as autologuos stem-stem cell transplantation and proteasome inhibition are limited by significant treatment-related toxicity. A novel target for PC depletion is CD38, a surface protein that is highly expressed on plasma cells (PCs) but also activated T-cells and most myeloid cells. Daratumumab is a monoclonal antibody targeting CD38 that is licensed for the treatment of multiple myeloma

Objectives: Here, we aimed to ascertain clinical safety and efficacy of Daratumumab for the treatment of refractory SLE, as well as to gain insights into effects of Daratumumab on the immune system.

Methods: We treated two SLE patients with life- and organ-threatening SLE with four weekly dosis of $16 \mathrm{mg} / \mathrm{kg}$ Daratumumab. We performed integrative analyses of clinical, serological and immunological effects over a follow-up period of 6 months. Using flow cytometry and single-cell RNA and T-cell receptor sequencing we followed CD38 expression and composition of peripheral blood leukocytes with a special focus on memory $T$ cells.

Results: Patient 1, a 50-year old woman, suffered from active biopsy-proven class III lupus nephritis (LN) with nephrotic syndrome, pericarditis, arthritis and skin rash. Upon Daratumumab treatment, her glomerular filtration rate normalized within 3 months and proteinuria gradually declined from 6.4 to $1.9 \mathrm{~g} / \mathrm{g}$ Creatinine during the 180-day follow-up period. Pericarditis, arthritis and skin rash completely resolved. Patient 2 , a 32-year-old woman, presented with autoimmune hemolytic anemia requiring blood transfusions, immune thrombocytopenia and cutaneous vasculitis. Her direct antiglobulin test normalized within 3 months and remained negative throughout follow-up with consecutive recovery of the hemolytic anemia. Immune thrombocytopenia stabilized and vasculitic skin lesions completely resolved. Infusions were well tolerated without severe adverse drug reactions. NK cells and Dendritic Cells were transiently depleted, while numbers of T cells, B cells and Monocytes in the peripheral blood remained stable. CD38+ memory $T$ cells that were expanded prior to treatment were virtually undetectable early after treatment. Their single cell transcriptomics demonstrated an upregulation of genes associated with activation, cytotoxicity and type 1 interferon response. CD38+ CD8+ memory T-cells showed marked oligoclonality. These prominent clones persisted upon treatment but their transcription profile gradually normalized.

Conclusion: Daratumumab appears to be a safe and effective treatment for refractory SLE. Further investigations are warranted to establish the efficacy in a clinical trial and to gain further insights into the pathophysiologic mechanism of action.

Disclosure of Interests: Lennard Ostendorf: None declared, Udo Schneider: None declared, Marie Urbicht: None declared, Philipp Enghard: None declared, Frederik Heinrich: None declared, Pawel Durek: None declared, Gitta Heinz: None declared, Henrik Mei: None declared, Mir-Farzin Mashreghi: None declared, Gerd Rüdiger Burmester Consultant of: AbbVie Inc, Eli Lilly, Gilead, Janssen, Merck, Roche, Pfizer, and UCB Pharma, Speakers bureau: AbbVie Inc, Eli Lilly, Gilead, Janssen, Merck, Roche, Pfizer, and UCB Pharma, Andreas Radbruch: None declared, Falk Hiepe: None declared, Tobias Alexander: None declared

DOI: 10.1136/annrheumdis-2020-eular.5052

\section{AB0386 \\ RITUXIMAB IN LUPUS NEPHRITIS. REAL CLINICAL PRACTICE}

C. Ramos Giráldez ${ }^{1}$, M. L. Velloso Feijoo ${ }^{2}$, S. Rodríguez Montero², J. L. Marenco ${ }^{2} .{ }^{1}$ Hospital Valme, Rheumatology Unit, Valme Hospital, Seville, Spain; ${ }^{1}$ Hospital Valme, Rheumatology Unit, Valme Hospital, Seville, Spain

Background: Lupus nephritis (LN) is a potentially life-threatening complication of systemic lupus erythematosus (SLE). Standard treatment of this entity includes glucocorticoids (GC) and immunosuppressants such as cyclophosphamide (CF), with remission rates between 20 and $70 \%$. Given its pathogenesis, the anti-CD20 antibody rituximab (RTX) may be useful, although the information available in the scientific literature remains controversial.
Objectives: To evaluate the effectiveness and safety of RTX in NL refractory to conventional treatment, under real clinical practice conditions.

Methods: Retrospective descriptive observational study, which included consecutive patients attended in a Rheumatology Unit who met SLICC 2012 criteria for SLE, with renal involvement, and who had received RTX for NL refractory to standard treatment. We collected different epidemiological, clinical (including other manifestations of SLE as well as previous treatments), analytical, RTX-related and adverse event variables. In all cases, renal function prior to the initiation of RTX was determined, as well as the degree of proteinuria and sediment alterations. The therapeutic response was evaluated considering partial response if they presented reduction of proteinuria in $>50 \%$ compared to initial and stabilization of renal function $( \pm 25 \%)$ or improvement of serum creatinine with respect to initial values; and complete response if they presented levels of serum creatinine $<1.2 \mathrm{mg} / \mathrm{dL}$ (or decrease to initial values or $\pm 15 \%$ of the basal value in those with creatinine $\geq 1,2 \mathrm{mg} / \mathrm{dL}$ ), proteinuria $\leq 0,5 \mathrm{~g} / 24 \mathrm{~h}$ and inactive sediment. A quantitative descriptive analysis has been carried out with the statistical program IBM SPSS 24.0 for Windows.

Results: Thirteen patients (11 women and 2 men) were included, with a mean age of 32.3 years and a mean follow-up time from the start of RTX of 11.09 years. Class IV NL was the most frequent (46.15\%), followed by Class III NL (38.46\%) Class V NL represented $7.69 \%$ and another $7.69 \%$ the combination of Class III+V. Renal failure was present in $46.2 \%$ of patients at the beginning of RTX, $84.6 \%$ hematuria and all patients presented proteinuria, of which $76.9 \%$ were confirmed to have nephrotic range and $84.6 \%$ showed hypoalbuminemia. With regard to previous treatments, all cases had received high-dose of GC and at least one immunosuppressant: $92.3 \%$ had failed to CF, a similar percentage had received azathioprine and $46.2 \%$ had failed to mycophenolate. After treatment with RTX, partial or complete response was achieved in $84.61 \%$, infections were identified in $46.15 \%$ of patients and allergic reactions in $15.38 \%$; most adverse events were mild and all cases developed favorably.

Conclusion: RTX treatment is effective in SLE and specifically in LN. More than $80 \%$ of patients in our study with refractory LN benefited from RTX treatment. Despite the not insignificant incidence of adverse events, most were mild and resolved without sequels or complications, so we can conclude that the safety of RTX is acceptable and should be considered as a treatment option for these patients according to the good risk-benefit profile.

Disclosure of Interests: Consuelo Ramos Giráldez: None declared, Maria Luisa Velloso Feijoo: None declared, Sergio Rodríguez Montero: None declared, Jose Luis Marenco Speakers bureau: ABbvie, Pfzer, lilly

DOI: 10.1136/annrheumdis-2020-eular.4305

\section{AB0387 1 TREATMENT STATUS FOR OSTEOPOROSIS IN PATIENTS WITH SYSTEMIC LUPUS ERYTHEMATOSUS: CROSS-SECTIONAL ANALYSIS FROM A LUPUS REGISTRY OF NATIONWIDE INSTITUTIONS (LUNA)}

K. E. Sada ${ }^{1}$, K. Hayashi ${ }^{1}$, Y. Asano ${ }^{1}$, Y. Katayama ${ }^{1}$, S. Hiramatsu Asano ${ }^{1}$, K. Ohashi ${ }^{1}$, M. Morishita ${ }^{1}$, H. Watanabe ${ }^{1}$, M. Narazaki ${ }^{1}$, Y. Matsumoto ${ }^{1}$, N. Yajima ${ }^{2}$, R. Yoshimi ${ }^{3}$, Y. Shimojima ${ }^{4}$, S. Ono ${ }^{5}$, H. Kajiyama ${ }^{6}$, K. Ichinose ${ }^{7}$ S. Sato ${ }^{8}$, M. Fujiwara ${ }^{9}$, J. Wada ${ }^{1}{ }^{1}$ Okayama University Graduate School of Medicine Dentistry and Pharmaceutical Sciences, Department of Nephrology, Rheumatology, Endocrinology and Metabolism, Okayama, Japan; ${ }^{2}$ Showa University School of Medicine, Tokyo, Japan; ${ }^{3}$ Yokohama City University Graduate School of Medicine, Yokohama, Japan; ${ }^{4}$ Shinshu University School of Medicine, Matsumoto, Japan; ${ }^{5}$ Yokohama City University Medical Center, Yokohama, Japan; ${ }^{6}$ Saitama Medical University, Saitama, Japan; ${ }^{7}$ Graduate School of Biomedical Sciences, Nagasaki University, Nagasaki, Japan; ${ }^{8}$ Fukushima Medical University School of Medicine, Fukushima, Japan; ${ }^{9}$ Yokohama Rosai Hospital, Yokohama, Japan

Background: Osteoporosis is one of the most important adverse effects of glucocorticoids in patients with systemic lupus erythematosus (SLE). Because osteoporosis is accelerated by chronic kidney disease (CKD), more attention should be paid to the treatment for osteoporosis in SLE patients with CKD. Many treatment options for osteoporosis have emerged recently, but treatment status in patients with SLE is not elucidated.

Objectives: The purpose of this study is to elucidate the treatment status for osteoporosis in patients with SLE among the CKD stages.

Methods: Using data from lupus registry of nationwide institutions (LUNA), a cross-sectional analysis was performed. We firstly described treatment status for osteoporosis in all enrolled patients. Secondary, treatment status for osteoporosis was compared among CKD stages. Finally, bone damage in Systemic Lupus International Collaborating Clinics/American College of Rheumatology Damage Index (SDI) was compared among CKD stages.

Results: The median age (interquartile range [IQR]) of enrolled 917 patients was 44 (34- 57) years and 809 patients (88\%) were female. CKD stages were follows: CKD stage 1, 234 (26\%); CKD stage 2, 465 (51\%); CKD stage 3, $189(21 \%)$ CKD stage 4, 9 (1\%); CKD stage 5, 16 (2\%). Median (IQR) age, female sex 
and median (IQR) previous maximum dose of prednisolone in patients with and without CKD ( $\geq$ CKD stage 3) were 56 (46.5-66) and 41 (32-50), 191 (89\%) and $615(88 \%)$, and $40(30-60)$ and 40 (30-55) mg/day, respectively. Bisphosphonate was administered in 388 (42\%) patients, vitamin D supplements in 448 (49\%), Ca supplements in $36(4 \%)$, denosumab in $20(2 \%)$ and teriparatide in $14(2 \%)$, respectively. Of enrolled patients, any treatment for osteoporosis was not administered in $226(25 \%)$ patients. In spite of more frequent bone damage in patients with CKD compared to those without CKD (15\% vs $10 \%, p=0.036)$, treatment status did not differ between patients with and without CKD (bisphosphonate: $41 \%$ vs $46 \%, p=0.29$; vitamin D supplements: $50 \%$ vs $44 \%, p=0.14$ ).

Conclusion: About a quarter of patients with SLE did not take any treatment for osteoporosis. Treatment for osteoporosis might be strengthened to prevent bone damage in SLE patients with CKD.

Disclosure of Interests: KEN-EI SADA Speakers bureau: I received speaker's fee from GSK and Astra Zeneca K.K., Keigo Hayashi: None declared, Yosuke ASANO: None declared, Yu Katayama: None declared, Sumie Hiramatsu Asano: None declared, Keiji Ohashi: None declared, Michiko Morishita: None declared, Haruki Watanabe: None declared, Mariko Narazaki: None declared, Yoshinori Matsumoto: None declared, Nobuyuki Yajima: None declared, Ryusuke Yoshimi: None declared, Yasuhiro Shimojima: None declared, Shigeru Ono: None declared, Hiroshi Kajiyama: None declared, Kunihiro Ichinose: None declared, Shuzo Sato: None declared, Michio Fujiwara: None declared, Jun Wada: None declared

DOI: 10.1136/annrheumdis-2020-eular.1202

\section{$\mathrm{AB0388}$ \\ IMPROVEMENT OF PATIENT-REPORTED OUTCOMES IN PATIENTS WITH PRIMARY SJÖGREN'S SYNDROME UNDERGOING CPAP-TREATMENT}

M. Seng ${ }^{1}$, B. C. Frye ${ }^{2}$, L. Vollmer ${ }^{2}$, F. Kollert ${ }^{3}$, R. Voll ${ }^{4}$, S. Finzel ${ }^{5} .{ }^{1}$ Medical Centre - University of Freiburg, Faculty of Medicine, Department of Rheumatology and Clinical Immunology, Medical Centre, 79106, Germany; ${ }^{2}$ Medical Centre - University of Freiburg, Faculty of Medicine, Department of Pneumology, Freiburg, Germany; ${ }^{3}$ University Hospital Bern, Department of Rheumatology, Immunology, and Allergology, Bern, Switzerland; ${ }^{4}$ Medical Centre - University of Freiburg, Faculty of Medicine, Department of Rheumatology and Clinical Immunology, Medical Centre, Freiburg im Breisgau, Germany; ${ }^{5}$ Medical Centre - University of Freiburg, Faculty of Medicine, Department of Rheumatology and Clinical Immunology, Medical Centre, Freiburg, Germany

Background: Fatigue is a frequent symptom in primary Sjögren's Syndrome (pSS) determining health-related quality of life in many patients. Earlier studies could show that sleep apnea syndrome (SAS) is associated with patient-reported outcomes in pSS-patients [1].

Objectives: To investigate whether treatment of an underlying SAS improves disease activity in pSS-patients.

Methods: 14 female pSS-patients from our rheumatology outpatient clinic were enrolled and screened for SAS; continuous positive airway pressure (CPAP)-treatment was initiated in patients with a sleep apnea/hypopnea index $>5 / h$ and a respiratory disturbance index $>15 / \mathrm{h}$ sleeping time (pSS treated with CPAP: pSS+; pSS untreated: pSS-). As controls, 11 SAS-patients (4 female) scheduled for initiation of CPAP-treatment were recruited from pulmonology department. In pSS, ESSDAI, ESSPRI, pain and patient global health on a visual analogue scale from 0-100mm were recorded. In both groups CRP, ESR, depression (PHQ-9), fatigue (FACIT), daytime sleepiness (EPS), and salivary gland ultrasound (SGUS) of the parotid and submandibular glands (PG; SMG) were obtained [2]. Data were collected at baseline (BL) and month 3 (M3). A p-value $<0.05$ was deemed significant.

Results: 6 pSS-patients were diagnosed with SAS and underwent CPAP-initiation, as well as all SAS-patients. Table 1 shows means \pm SD of all assessments. EPS indicated normal sleep propensity in all groups at both time points. Fatigue was severe in all groups at BL. Numerically in SAS and pSS+, fatigue and depression trended towards improvement between $\mathrm{BL}$ and M3. Depression scores were moderate in all groups at BL; in SAS, PHQ-9 score showed a trend towards improvement at M3. SGUS grades 0 or 1 (i.e. normal or unspecific SGUS pattern) were found in $46.43 \%$ of $P G$ and SMG at BL, in $40.39 \%$ at $M 3$ in pSS, and in $100 \%$ in SAS at both time points. Grades 2 or 3 (pSS-typical US-pattern or gland destruction) were exclusively found in pSS at BL (53.57\%) and M3 (59.62\%). pSS-SGUS scores worsened irrespective of CPAP-treatment. Conclusion: Even though this is only preliminary data of a small fraction of patients who completed M3 so far, there is already a clear tendency towards reduced fatigue, inflammatory markers and depression both in the CPAP-treated pSS and in the SAS-patients compared to the non-CPAP-treated pSS-patients. References:

[1] Usmani ZM Sleep Medicine 2012; 2. Jousse-Joulin S Ann Rheum Dis. 2019

\begin{tabular}{|c|c|c|c|c|}
\hline & & Baseline (mean $\pm S D)$ & $\begin{array}{c}\text { Month } 3 \\
(\text { mean } \pm S D)\end{array}$ & p-Values \\
\hline \multirow[t]{3}{*}{ CRP } & pSS+ & $4.58 \pm 2.98$ & $2.79 \pm 0.56^{*}$ & ${ }^{*} \mathrm{p}=0.014$ \\
\hline & pSS- & $6.53 \pm 5.07$ & $4.08 \pm 3.69$ & \\
\hline & SAS & $4.27 \pm 1.80$ & $4.76 \pm 3.50^{*}$ & \\
\hline \multirow[t]{3}{*}{ ESR } & pSS+ & $30.50 \pm 21.92$ & $24.75 \pm 10.69^{+}$ & ${ }^{+} p=0.031$ \\
\hline & pSS- & $34.75 \pm 37.92$ & $47.29 \pm 37.82^{\wedge}$ & $\hat{p}=0.013$ \\
\hline & SAS & $20.67 \pm 17.56$ & $11.67 \pm 11.38^{+^{\wedge}}$ & \\
\hline \multirow[t]{2}{*}{ ESSDAI } & pSS+ & $10.67 \pm 4.97$ & $11.00 \pm 6.08$ & \\
\hline & pSS- & $11.63 \pm 3.25$ & $9.43 \pm 4.72$ & \\
\hline \multirow[t]{2}{*}{ ESSPRI } & pSS+ & $6.75 \pm 1.44 "$ & $6.47 \pm 1.75$ & " $p=0.037$ \\
\hline & pSS- & $5.18 \pm 1.32 "$ & $5.46 \pm 1.77$ & \\
\hline \multirow[t]{2}{*}{ VAS pain } & pSS+ & $55.83 \pm 21.61^{\circ}$ & $54.17 \pm 24.60$ & ${ }^{\circ} \mathrm{p}=0.037$ \\
\hline & pSS- & $27.50 \pm 26.33^{\circ}$ & $34.25 \pm 26.87$ & \\
\hline \multirow[t]{2}{*}{ VAS PGH } & pSS+ & $58.50 \pm 11.67$ & $56.00 \pm 19.54$ & \\
\hline & pSS- & $52.88 \pm 30.86$ & $53.00 \pm 23.05$ & \\
\hline \multirow[t]{3}{*}{ FACIT } & pSS+ & $24.00 \pm 6.03$ & $29.00 \pm 10.56^{*}$ & ${ }^{*} p=0.059$ \\
\hline & pSS- & $27.88 \pm 14.75$ & $30.50 \pm 12.54$ & \\
\hline & SAS & $29.36 \pm 10.46^{\Delta}$ & $37.82 \pm 10.73^{¥ \Delta}$ & ${ }^{\Delta} p=0.054$ \\
\hline \multirow[t]{3}{*}{ PHQ-9 } & pSS+ & $11.50 \pm 3.02$ & $9.33 \pm 3.88 \infty$ & \\
\hline & pSS- & $10.86 \pm 5.05$ & $9.00 \pm 2.51 \circledast$ & \\
\hline & SAS & $10.11 \pm 5.33 \times$ & $6.55 \pm 5.75 \times \circledast \infty$ & $\begin{array}{c}{ }^{\times} \mathrm{p}=0.059 ; \AA \mathrm{p}=0.068 ; \\
\infty \mathrm{p}=0.087\end{array}$ \\
\hline
\end{tabular}

Disclosure of Interests: Magdalena Seng: None declared, Björn Christian Frye: None declared, Lydia Vollmer: None declared, Florian Kollert Employee of: Novartis, Reinhard Voll: None declared, Stephanie Finzel: None declared DOI: 10.1136/annrheumdis-2020-eular.2020

\section{AB0389 GLUCOCORTICOID WITHDRAWAL IN SYSTEMIC LUPUS ERYTHEMATOSUS: ANALYSIS OF 750 SLE PATIENTS FROM THE RUSSIAN AND KYRGYZ COHORTS.}

S. Solovyev ${ }^{1}$, E. Aseeva ${ }^{1}$, G. Koilubaeva ${ }^{2}$, E. Nasonov ${ }^{1}$, A. Lila ${ }^{1}$, N. Nikishina ${ }^{1}$ ${ }^{1}$ V.A. Nasonova Research Institute of Rheumatology, Moscow, Russia, Intensive Care Department, Moscow, Russian Federation; ${ }^{2}$ National Center for Cardiology and Internal Medicine named after Academician M. Mirrakhimov, Rheumatology Department, Bishkek, Kyrgyzstan

\section{Background:}

Objectives: Glucocorticoids (GCs) have been the mainstream of systemic lupus erythematosus (SLE) treatment for the last 70 years. GCs allow to achieve effective control over SLE activity quite rapidly - both in mild and severe disease. The majority of SLE patients have received GC therapy; in some cohorts up to $80 \%-100 \%$ of patients continue on low maintenance GCs doses $<7.5 \mathrm{mg} /$ day for many years perhaps some of them are treated indefinitely. It is clear that cumulative GCs dose is responsible for adverse effects. But it remains still unclear whether GCs should be continued indefinitely and, if not, when and how this treatment should be discontinued. On the other hand, treat-to-target SLE recommendations suggest GC withdrawal where possible as an important target of the treatment plan.

Methods: Patients who attempted GCs withdrawal were included in the Eurasian SLE RENAISSANCE cohort. A retrospective analysis of 350 patients from Russia and 400 patients from Kyrgyzstan was conducted. The following information was assessed during withdrawal attempts: SLE duration, disease activity at the onset and initiation of GCs dose reduction, therapy at SLE onset, the duration of the last flare, activity and therapy at the end of FUP, and duration of remission after GCs withdrawal. Definitions of remission were applied to GCs withdrawal in line with European consensus criteria.

Results: Out of 750 patients with a follow-up of about 6 years (IQR 1-23), GCs withdrawal due to persistent remission was documented in 15 patients $(2.0 \%)$. In 14 out of these 15, SLE onset was associated with high disease activity based on SLEDAI $2 \mathrm{~K}>8$. High level of anti-DNA and a decrease in C3 $\backslash \mathrm{C} 4$ complement were present in 12, 4 patients had nephritis with preserved renal function, 4 patients manifested signs of CNS damage (convulsions, headaches, sleep disturbances, memory issues, neuropathy, hallucinations), and another 5 had vasculitis. 10 patients were administered pulse therapy with $3 \mathrm{~g}$ methylprednisolone due to high disease activity. Initiation of GCs dose reduction with intent to discontinue in 7 patients was substantiated by prolonged clinical remission, meanwhile SLE duration in this group varied from 2 to 20 years, and duration of the last flare - from 6 to 165 months. Acute onset with high disease activity reaching 12-23 scores by SLEDAI $2 \mathrm{~K}$ was documented in 8 cases of early SLE with disease duration varying from 1.5 to 6 months. These patients were prescribed the most aggressive induction therapy, including cascade plasma filtration in combination with pulse therapy, cyclophosphamide and Rituximab at $1 \mathrm{~g}$ dose. Remission (SLEDAI2K 0-2 scores) was achieved 4-6-8 months later after termination of aggressive induction therapy. The duration of remission after GCs withdrawal in all 15 patients ranged from 3.5 to 240 months. In 8 patients with aggressive induction therapy, remission lasted from 18 to 240 months. In 2 